\title{
Camera-based Online Flame-Stability-Monitor for Load-Flexible Biomass Co-Combustion
}

\author{
Jörg Matthes ${ }^{1}$, Patrick Waibel ${ }^{1}$, Markus Vogelbacher ${ }^{1}$, Hans-Joachim Gehrmann ${ }^{2}$, Hubert B. Keller ${ }^{1}$ \\ ${ }^{1}$ Institute for Automation and Applied Informatics, Karlsruhe Institute of Technology (KIT) \\ Hermann-von-Helmholtz-Platz 1, 76344 Eggenstein-Leopoldshafen, Germany \\ ${ }^{2}$ Institute for Technical Chemistry, Karlsruhe Institute of Technology (KIT) \\ Hermann-von-Helmholtz-Platz 1, 76344 Eggenstein-Leopoldshafen, Germany \\ Joerg.Matthes@kit.edu
}

\begin{abstract}
With an increasing share of fluctuating wind and solar power in the future energy system conventional combustion power plants have to enable a high load-flexibility. Additionally, a partial substitution of coal by biogenic fuels is desired. Especially in low load conditions a total shut down of a combustion plant has to be prevented. Instead, a stable low load operation has to be realized, e.g. by increasing the share of biogenic fuels with a lower calorific value, and thus decreasing the total power. Unfortunately, this usually leads to a destabilization of the flame, which - if detected - can partially be circumvented by adapting fuel-, air- and swirl settings of the burner system. In this paper, a new image processing system for the online flame stability monitoring based on a camera system in the visual spectral range is presented. The flame stability monitor is demonstrated at a pilot scale power plant with co-combustion of torrified coal with hard coal.
\end{abstract}

Keywords: Flame Stability Monitoring, Image Processing, Camera, Biomass Co-Combustion.

\section{Introduction}

The co-combustion of biogenic with fossil fuels in power plants is an important technology to reduce the fossil $\mathrm{CO}_{2^{-}}$ impact on electricity generation. The advantages are the sharing of existing facilities, the fuel flexibility, a wide range of usable fuels and achieving higher overall efficiency for electricity generation from biogenic fuels. Therefore, cocombustion in large thermal power plants can lead to an overall saving of fuels compared to independent fossil and biomass power plants [1-5]. With a higher share of wind and solar power in the future electrical power grid, thermal power plants must be able to deal with a highly load flexibility. Thus, load flexibility together with fuel flexibility in cocombustion power plants is a major goal of recent research.

At the Karlsruhe Institute of Technology (KIT), a pilot-scale power plant named BRENDA with a 1 MW multi-fuel burner and a $2.5 \mathrm{MW}$ boiler is used to investigate different fuel-mix and load scenarios. Especially experiments for cocombustion of biomass like straw and torrified (torr) coal with hard coal were performed for different burning parameters and load setups. During the experiments, the multi-fuel burner flame can be observed by two camera systems: a high-speed camera system with up to 2000 frames per second (fps) and a high dynamic range (HDR) camera system with 25 fps both in the visible spectral range. The algorithms for the detection of fuel particles and their trajectories within the flame based on the high-speed camera system are presented in [6].

In this article a new image processing system for the online analysis of the flame stability at different load and fuel scenarios is presented based on the HDR camera system. An algorithm for a robust detection of the burner flame within the camera images is described. Based on the segmented flame, the flame stability is calculated using a sequence of flame images.

\section{Pilot-scale Power Plant BRENDA}

The combustion chamber BRENDA (Fig.1) installed at the KIT is used to develop and investigate multi-fuel burner systems in conjunction with the development of new camera based burner analysis and control methods. The focus is on the co-firing of alternative fuels with pulverized coal [7]. 
To simulate a combustor with a base-load and fast-loaded process, a rotary kiln is used as a horizontal primary combustion chamber (PCC) with a thermal throughput of $1.5 \mathrm{MW}$. Connected to the PCC the vertical combustion chamber with the multi-fuel dust burner is installed. The PCC generates the base load with a constant flue gas flow generated from a combination of an oil/gas burner and solid fuels (Fig.1).

The combustion chamber has a diameter of $1.9 \mathrm{~m}$ and is $15 \mathrm{~m}$ high. The pulverized-multi-fuel burner is located in the middle of the combustion chamber. The dust burner has a thermal power of 1.0 MW at a maximum fuel flow of about $100 \mathrm{~kg} / \mathrm{h}$.

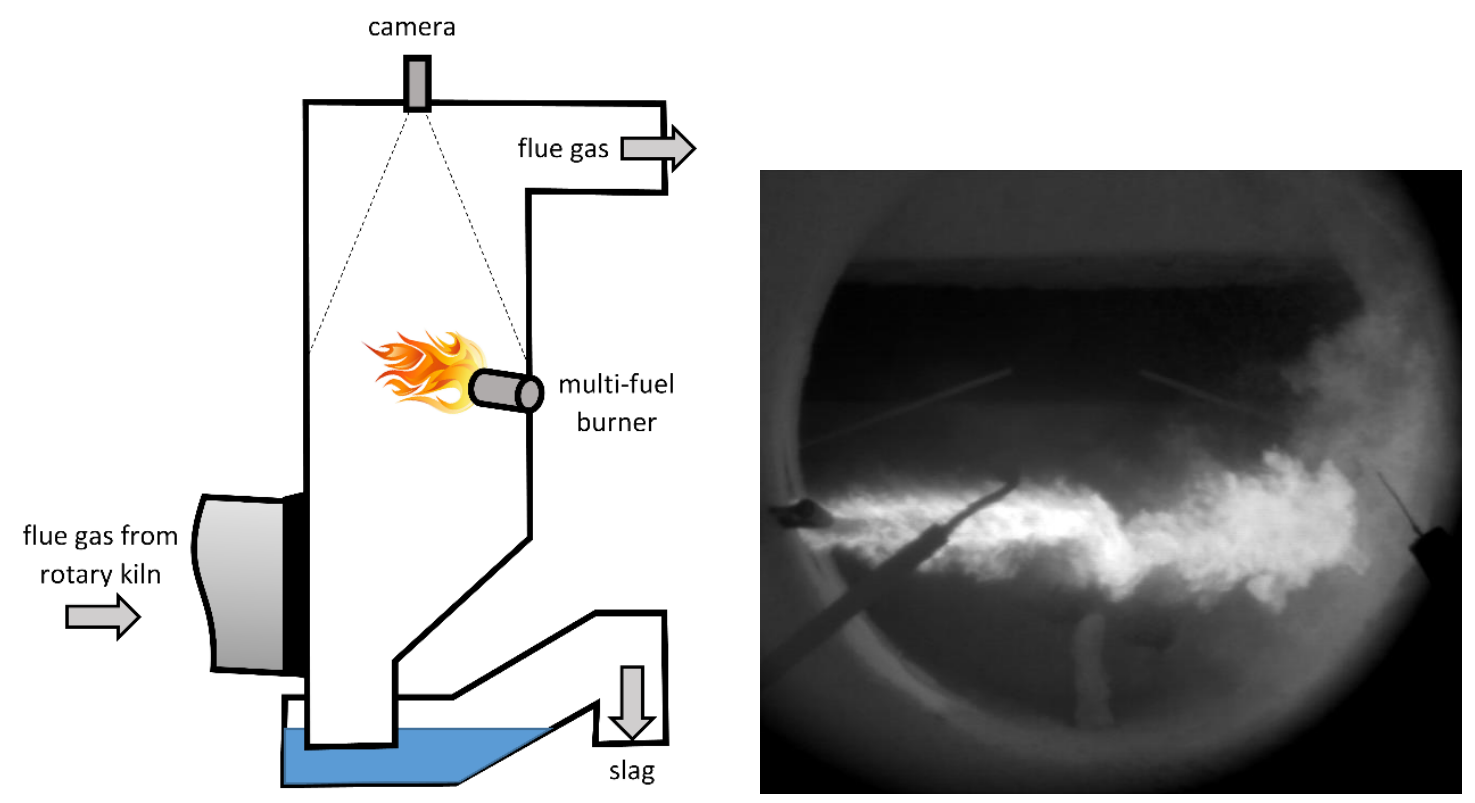

Fig. 1: Setup of experimental combustion chamber BRENDA and camera image of the flame.

The dust burner is equipped with an integrated swirl generator. The swirl of the combustion air can be adjusted manually. The alternative fuels may either be added to the coal flow or fed into the burner separately via a central lance. The hot flue gases leaving the combustion chamber enter the boiler where they are cooled down to approximately $300{ }^{\circ} \mathrm{C}$ and generate saturated steam of 40 bar and $250{ }^{\circ} \mathrm{C}$. The boiler has a maximum capacity of 2.5 MW. Flue gas cleaning meets the requirements outlined in the 17th Federal Emission Control Ordinance and mainly consists of a spray dryer, a fabric filter, two scrubbers, and an SCR catalyst [7]. An opening in the ceiling of the combustion chamber allows for the insertion of a water-cooled camera probe. A high-dynamic-range (HDR) camera system is used for capturing grayscale images of the multi-fuel burner flame at 25 frames per second. The image resolution is $768 \times 578$ pixels. This corresponds to the size of a pixel of approx. $3.4 \times 3.4 \mathrm{~mm}$ in the examined burner level. Fig. 2 shows a single image of the burner flame. The bright diffuse areas in the image indicate the radiation of burning hard and torrified (torr) coal dust from biomass. In addition, the burning torr coal particles can be seen as bright points over the entire cross section of the combustion chamber. The median particle size D50 of the torr coal is about $650 \mu \mathrm{m}$ and $100 \mu \mathrm{m}$ for the hard coal. The maximum particle size of the torr coal is about $3000 \mu \mathrm{m}$.

\section{Image Processing System}

The aim of the image processing system is to detect the flame (segmentation of input image) and calculate parameters that describe the properties of the current flame e.g. the flame stability. The flame detection is performed with a dynamic gray value threshold. For each input image, a threshold is determined, which allows a segmentation of the input image into foreground (bright flame) and background (dark combustion chamber). 
A standard procedure to find such a threshold is Otsu's method [8], which tries to find a threshold value $t_{\text {OTsu }}$, that minimizes the weighted sum of the intra-class variances of the foreground and background. That means, it divides the input image into two homogeneous regions as good as possible.

In average conditions of the combustion chamber, Otsu's method leads to satisfying flame detections as can be seen in Fig. 3 (left). Otsu's method is applied to the region of interest (ROI, blue line in Fig. 3) and yields the flame region (red line in Fig. 3). Here, only the largest connected region is used as flame detection. Additionally, morphological operations (opening and closing) [9] are applied to get a compact flame region without holes and artefacts.
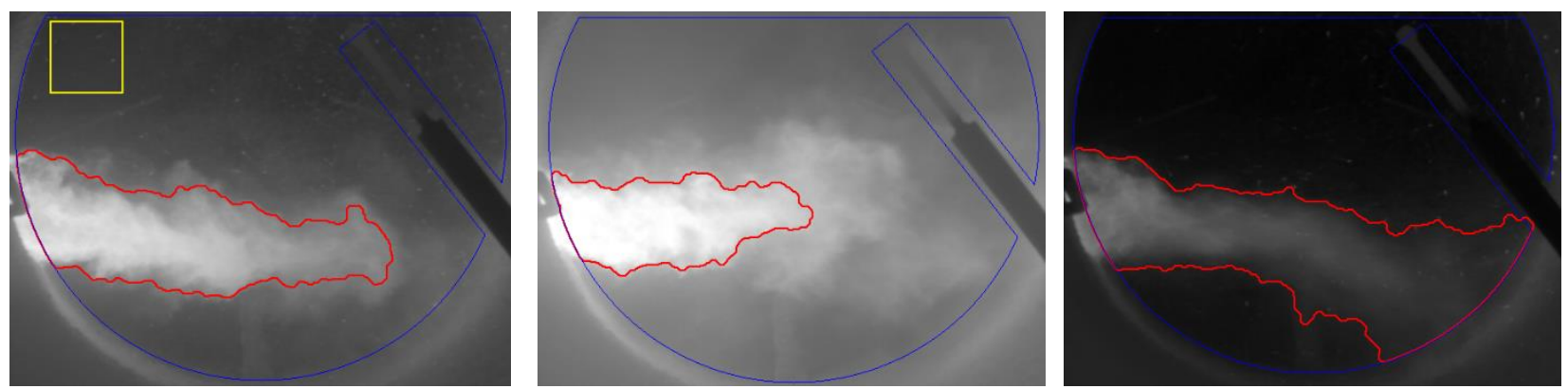

Fig. 3: Otsu based flame detection (red: segmented flame, blue: region of interest, yellow: reference field) for average conditions (left), hot conditions (center) and cold conditions (right) of the combustion chamber.

Unfortunately, in hot conditions of the combustion chamber Otsu's method tends to result in thresholds, which are too high and thus yield flame detections, which are too small as shown in Fig. 3 (center). Similarly, cold conditions of the combustion chamber result in Otsu thresholds, which are too low and thus yielding flame detections, that are too big (Fig. 3 right). To improve the flame detection, a threshold correction is introduced, which is based on the mean grayvalue within a reference field $\mathrm{g}_{\text {ref }}$ (Fig. 3 yellow) in a region of the ROI that is always part of the background. In our examples, for average conditions $\mathrm{g}_{\text {ref }}$ is approx. 50, for hot conditions up to 100 and for cold conditions approx. 0 .

For the flame detection, a modification of the Otsu threshold by a correction term is proposed:

$$
t_{\text {mod }}=t_{\text {OTSU }}+0.5 \cdot\left(50-g_{\text {ref }}\right)
$$

Thus, in average conditions with $\mathrm{g}_{\text {ref }} \approx 50$ the original Otsu threshold is used. In hot conditions $\left(\mathrm{g}_{\text {ref }}>50\right)$ the threshold is decreased, and in cold conditions $\left(\mathrm{g}_{\mathrm{ref}}<50\right)$ it is increased. The improved flame detections with the modified threshold are shown for normal, hot and cold conditions in Fig. 4.
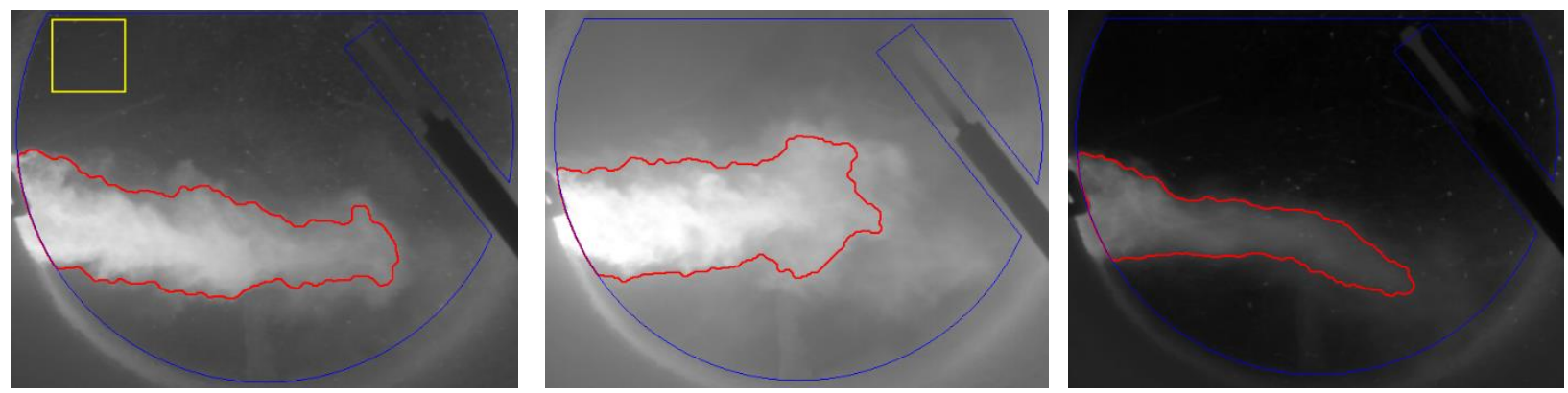

Fig. 4: Flame detections with modified Otsu threshold for average conditions (left), hot conditions (center) and cold conditions (right) of the combustion chamber. 
After the detection of the flame for each input image, the flame stability can be calculated based on a sequence of flame detections. For the flame stability, the intersection area (stable part) of all flame detections for a given sequence (i.e. 25 frames over $1 \mathrm{sec}$ ) is calculated. Thus, the fluctuation of the flame area as well as of the flame position is taken into account for the flame stability measure. Fig. 5 shows the evolution of the stable part (green) for 4 different image sequences with decreasing flame stability from (a) to (d). Each row represents a sequence of 25 frames from which 4 frames are depicted. For the flame stability calculation for each sequence, frame no. 25 in Fig. 5 is relevant.

Frame no. 1

a)

b)

c)

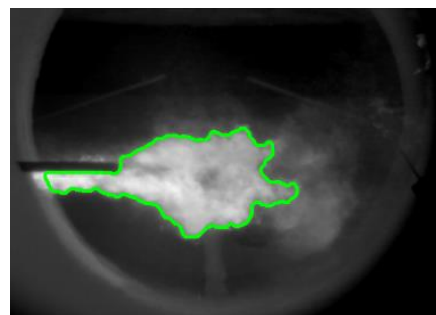

d)

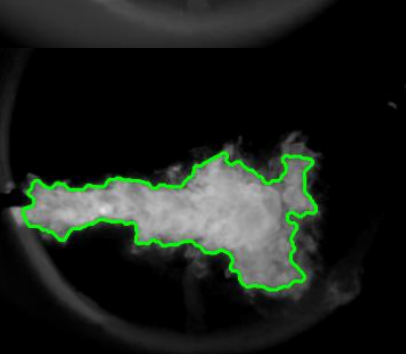

)

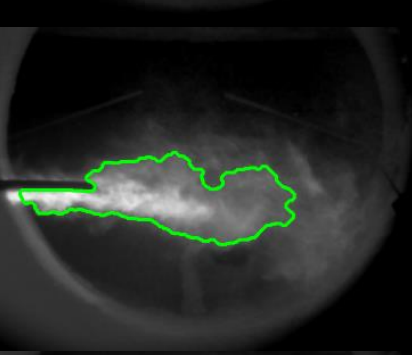

(1)

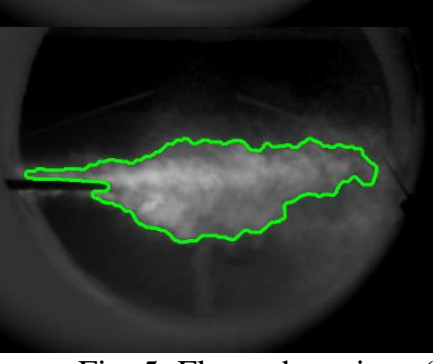

Fig. 5: Flame detections (red)
8

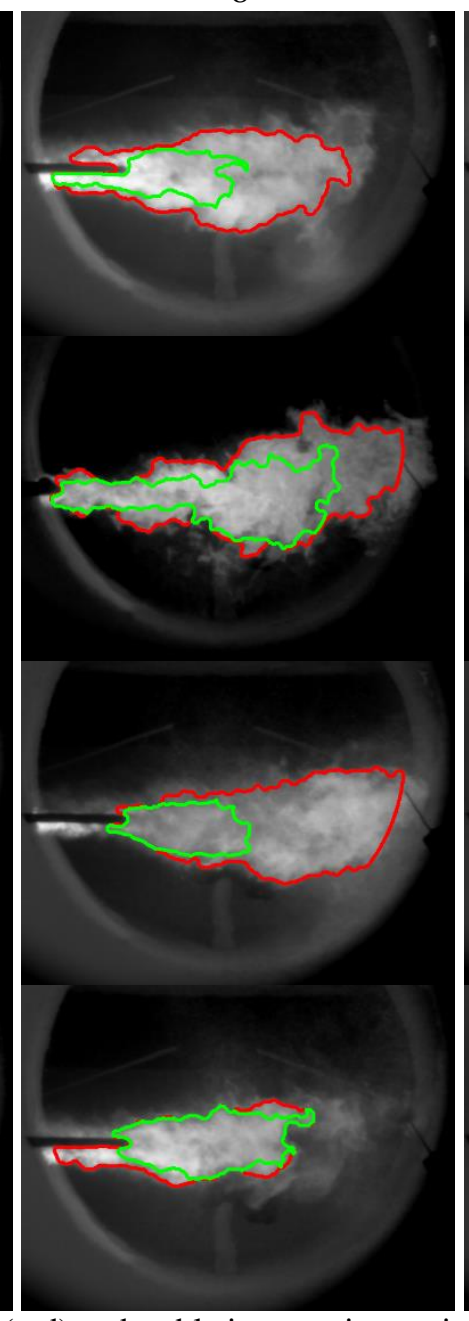

16

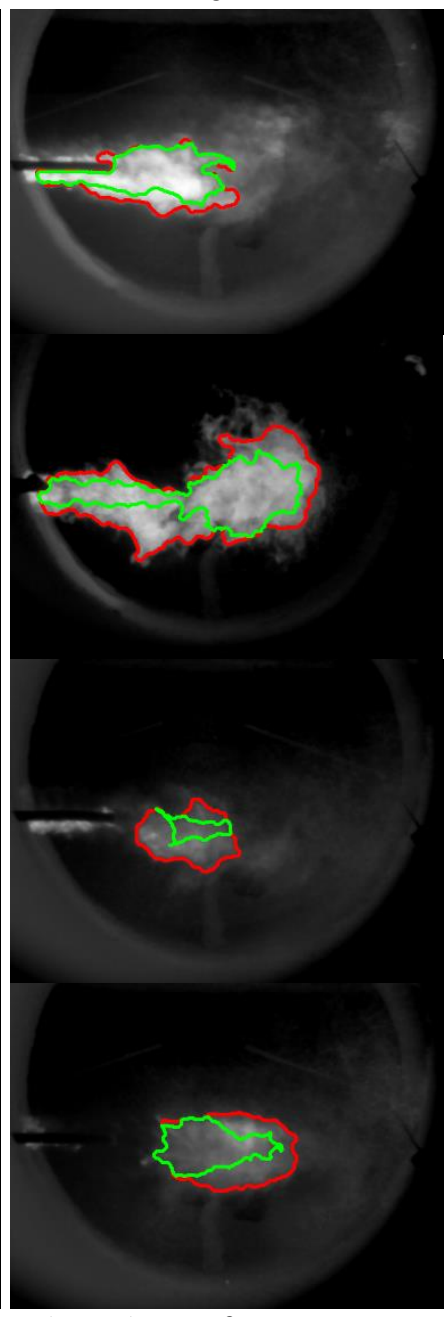

25

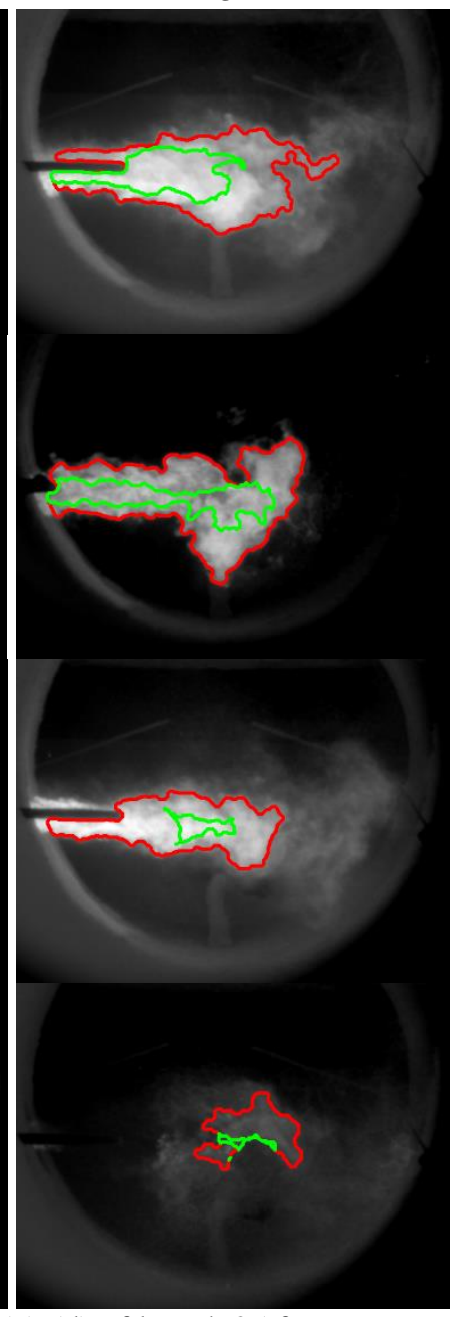

with decreasing flame stability from (a) to (d).

By implementing the determination of the stable flame part as a moving filter, for each frame the current flame area $A_{\text {current }}$ and the stable area $A_{\text {stable }}$ can be calculated online. The flame stability for each frame is then calculated by

$$
\text { flame stability }=\frac{A_{\text {stable }}}{A_{\text {current }}} \cdot 100 \%
$$


In Fig. 5 the stable part of the flame is depicted in green. The ratio of the area of the green region and the area of the red region yields the flame stability. It has to be mentioned, that since there is no clear physical boundary of the flame, the image based flame detection is only an estimation. Thus, the flame stability calculation can never be an exact measurement, but it gives a good rating of the flame stability.

\section{Experimental Results}

Here, the results of the camera-based observation of two experiments are presented (Fig. 6 and Fig. 7). Both experiments aim to investigate the power flexibility of the multi-fuel burner system especially for the case of low load conditions. In both experiments, the overall thermal power is reduced by decreasing the hard coal mass flow and simultaneously increasing the torr coal mass flow until the flame becomes totally unstable. Then hard coal is increased again while decreasing the torr coal mass flow thus increasing the overall thermal power again. In experiment 1 the torr coal is fed by the central lance and the hard coal is fed by the annular gap of the burner, while in experiment 2 the torr coal is fed by the annular gap and the hard coal is fed by the central lance.

In experiment 1 (Fig. 6), the power decrease starts at 10:40 which leads to a decrease of the camera based flame stability. At 10:46 a local minimum of the flame stability is reached. This matches also the human evaluation of the flame stability by the operators. Interestingly, the flame stability temporarily increases at 10:47:30, although the hard coal mass flow is further decreased. The operators also confirmed this camera-based observation. Due to technical reasons at a certain level, the hard coal flow cannot be decreased further but drops to zero (10:48). This leads to an immediate drop of the flame stability. The abrupt increase of hard coal at 10:51 leads to a rapid increase of the flame stability but then the same effect as at 10:46 can be observed. The flame stability temporarily decreases and reaches a local minimum although the hard coal mass flow increases. Not before 10:53:30 the flame stability increases with increasing hard coal mass flow and overall power.

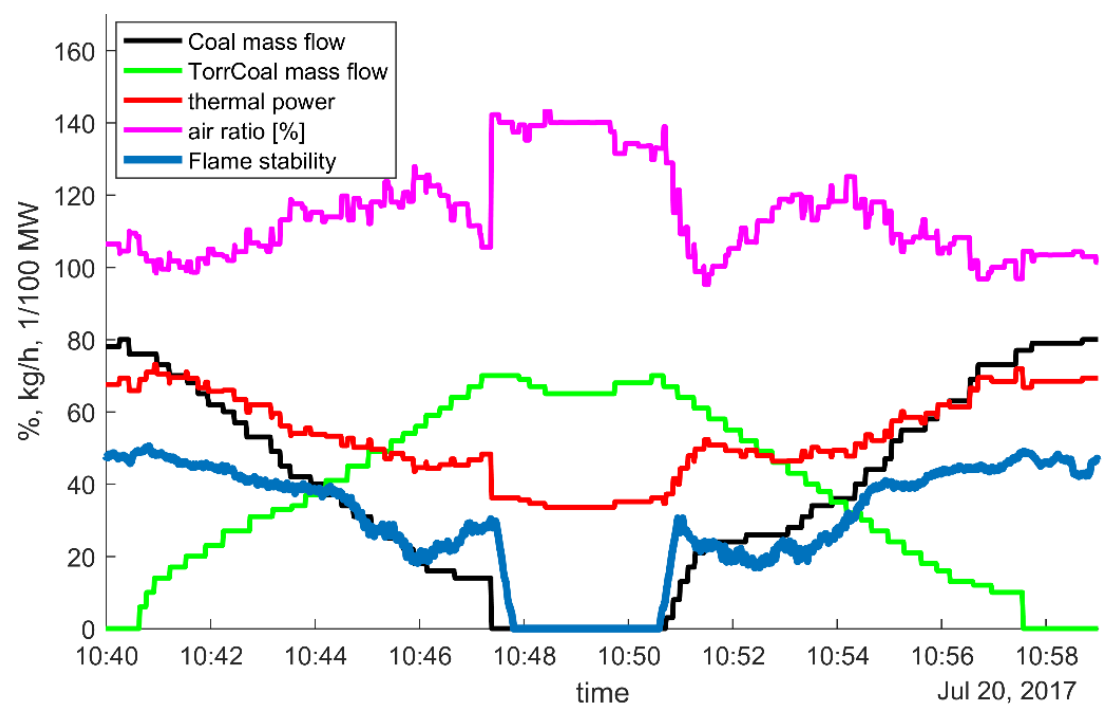

Fig. 6: Courses of hard and torr coal mass flow, calculative thermal power, air ratio and camera based calculated flame stability for experiment 1.

A similar behavior as in experiment 1 can also be observed in experiment 2 (Fig. 7). Again, temporary minima of the flame stability arise at 16:08 and 16:17. Apparently, there is no monotonic relation between hard coal mass flow and flame stability. This means, that the switched feedings (annular gap or central lance) of fuels torr coal and hard coal does not change the flame stability behavior. Comparing the air ratio with the flame stability in the two experiments, both signals appear almost mirrored. This means, that the closer the air ratio to $100 \%$, i.e. the lower the air ratio, the more stable is the observed flame. This holds up to a minimum amount of hard coal. Under a certain hard coal mass flow the flame becomes 
instable independently of the air ratio. It is assumed, that with higher shares of torrified coal, the three times greater particle size of the torrified coal, which correlates to a reduced specific surface of the particles for heat and mass leads to the flame instability.

\section{Conclusion}

In this article a new online flame stability monitoring system was presented. It is based on a HDR camera system in the visual spectral range. First, an algorithm for a robust detection of the burner flame within the camera images is described. Then a flame stability measure is presented, that is calculated based on the stable flame region in a sequence of flame images.

In the experiments at a pilot-scale combustion power plant with co-combustion of torrified coal with hard coal it is shown, that the camera based calculated flame stability measure matches the human impression by the plant operators. The experiments showed, that in low load conditions of the plant the flame stability decreases with decreasing hard coal mass flow. These first measurements demonstrate that in low load operation a certain amount of hard coal is necessary to keep the flame stable. Interestingly, the relation is not monotonic, but it also depends on the air ratio, which will be investigated in further experiments more in detail. Prospective, a camera based control system is to be developed that will automatically adapt the burner settings to stabilize the flame based on the camera based online flame monitor.

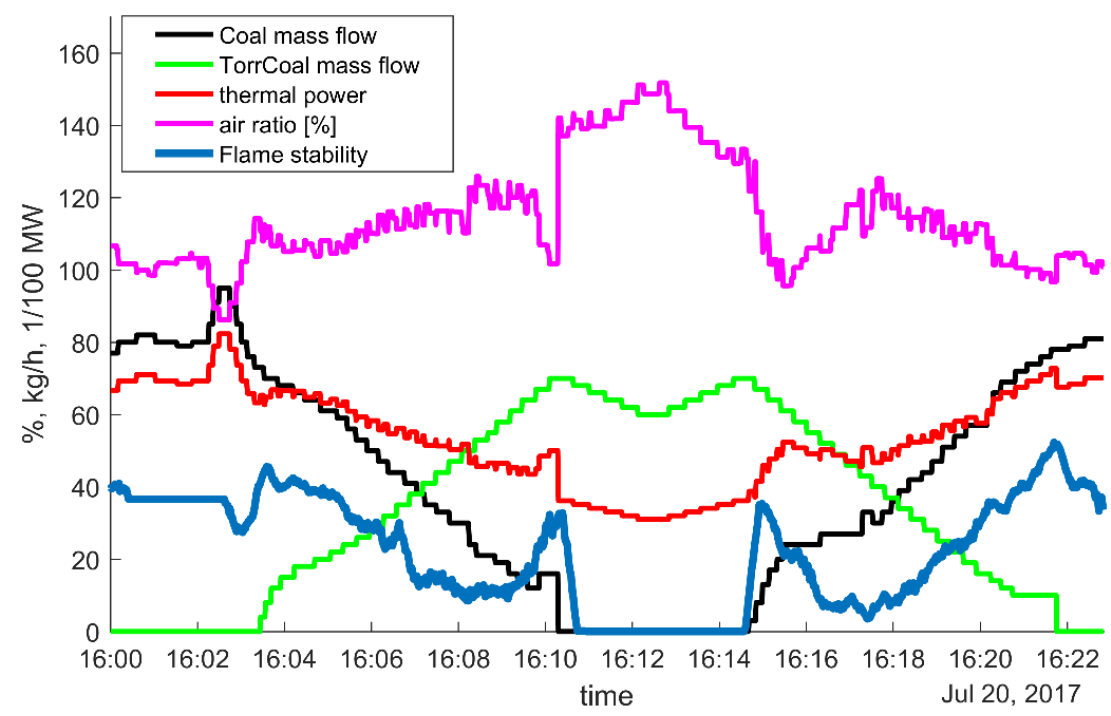

Fig. 7: Courses of hard and torr coal mass flow, calculative thermal power, air ratio and camera based calculated flame stability for experiment 2.

\section{Acknowledgements}

This work was supported by the Helmholtz long-range strategic research funding (EMR, POF III).

\section{References}

[1] VGB POWERTECH, Advantages and Limitations of Biomass Co-combustion in Fossil Fired Power Plants, 2008.

[2] C. Yin, S. K. Kær, L. Rosendahl and S. L. Hvid, "Co-firing straw with coal in a swirl-stabilized dual-feed burner: Modelling and experimental validation,” Bioresource Technology, vol. 101, no. 11, pp. 4169-4178, 2010.

[3] S. van Loo and J. Koppejan. The handbook of biomass combustion and co-firing. Earthscan, London and Sterling and VA, 2008. 
[4] C. Henderson, Increasing the flexibility of coal-fired power plants. IEA Clean Coal Centre, 2014.

[5] M. Cremers, E. Pfeiffer, T. Konings and R. Meijer, "Co-firing high percentages - new chances for existing power plants," in IEA tasks 32. Workshop, KEMA, 2008.

[6] J. Matthes, J. Hock, P. Waibel, A. Scherrmann, H.-J. Gehrmann and H. B. Keller, "A high-speed camera based approach for the on-line analysis of particles in multi-fuel burner flames," Experimental Thermal and Fluid Science vol. 73, pp. 10-17, 2016.

[7] H.-J. Gehrmann, T. Kolb, H. Seifert, P. Waibel, J. Matthes, H. Keller and T. Leibfried, "Co-combustion of low rank fuels in power plants," in 19th European Biomass Conference and Exhibition, pp. 1334-1337, 2011.

[8] N. Otsu, "A Threshold Selection Method from Gray-Level Histograms," in IEEE Transactions on Systems, Man and Cybernetics vol. 9, no. 1, S. 62-66, 1979.

[9] L. Vincent, "Morphological grayscale reconstruction in image analysis: Applications and efficient algorithms," IEEE Transactions on Image Processing, vol. 2, pp. 176-201, 1993. 